\title{
Antiskyrmions and their electrical footprint in crystalline mesoscale structures
}

\section{Moritz Winter}

Francisco J. T. Goncalves

Ivan Soldatov

Yangkun He

Belén Zéuniga Céspedes

Peter Milde

Kilian Lenz

Sandra Hamann

Marc Uhlarz

Praveen Vir

Markus König

Philip J. W. Moll

Richard Schlitz

Sebastian T. B. Goennenwein

Lukas M. Eng

Rudolf Schaefer

Joachim Wosnitza

Claudia Felser

Jacob Gayles

Toni Helm ( $\nabla$ t.helm@hzdr.de )

Helmholtz-Zentrum Dresden-Rossendorf https://orcid.org/0000-0002-0938-8537

\section{Research Article}

Keywords: skyrmions, antiskyrmions, skyrmionics, topological Hall effect, anomalous Hall effect, magnetotransport, atomistic spin-dynamic simulations, magnetooptical Kerr effect microscopy, magnetic force microscopy, focused ion beam, microfabrication, racetracks, mesoscale structures, ferromagnetic resonance, magnetic anisotropy

Posted Date: November 4th, 2021

DOI: https://doi.org/10.21203/rs.3.rs-1047981/v1

License: (c) (i) This work is licensed under a Creative Commons Attribution 4.0 International License.

Read Full License 



\title{
Antiskyrmions and their electrical footprint in crystalline mesoscale structures
}

\author{
M. Winter ${ }^{1,2}$, F. J. T. Goncalves ${ }^{3}$, I. Soldatov ${ }^{4,5}$, Y. He ${ }^{2}$, B. E. Zúñiga Céspedes ${ }^{2,6}$, \\ P. Milde ${ }^{6}$, K. Lenz ${ }^{3}$, S. Hamann ${ }^{1}$, M. Uhlarz ${ }^{1}$, P. Vir ${ }^{2}$, M. König ${ }^{2}$, P. J. W. Moll ${ }^{2,7}$, \\ R. Schlitz ${ }^{8}$, S. T. B. Goennenwein ${ }^{8}$, L. M. Eng ${ }^{6,10}$, R. Schäfer ${ }^{4,8}$, J. Wosnitza ${ }^{1,8,10}$, \\ C. Felser ${ }^{2,10}$, J. Gayles ${ }^{2,9}$, and T. Helm ${ }^{1,2, *}$
}

${ }^{1}$ Dresden High Magnetic Field Laboratory (HLD-EMFL), Helmholtz-Zentrum Dresden-Rossendorf, 01328 Dresden,Germany

${ }^{2}$ Max Planck Institute for Chemical Physics of Solids, 01187 Dresden, Germany, ${ }^{3}$ Institute of Ion Beam Physics and Materials Research, Helmholtz-Zentrum Dresden-Rossendorf, 01328 Dresden, Germany.

${ }^{4}$ Leibniz Institute for Solid State and Materials Research Dresden, 01069 Dresden, Germany

${ }^{5}$ Institute of Natural Sciences, Ural Federal University, 620002 Ekaterinburg, Russia.

${ }^{6}$ Institute of Applied Physics, Technical University Dresden, 01062 Dresden, Germany.

${ }^{7}$ École Polytechnique Fédérale de Lausanne, 1015 Lausanne, Switzerland.

${ }^{8}$ Institute for Material Science, Technical University Dresden, 01062 Dresden, Germany

${ }^{9}$ Department of Physics, University of South Florida, Tampa, Florida 33620, USA.

${ }^{10}$ Würzburg-Dresden Cluster of Excellence ct.qmat, 01062 Dresden, Germany.

*E-mail: t.helm@hzdr.de

(Dated: October 11, 2021) 


\begin{abstract}
Skyrmionics materials hold the potential for future information technologies, such as racetrack memories. Key to that advancement are skyrmionics systems that exhibit high tunability and scalability, with stored information being easy to read and write by means of all-electrical techniques. Topological magnetic excitations, such as skyrmions and antiskyrmions give rise to a characteristic topological Hall effect (THE) in electrical transport. However, an unambiguous transport signature of antiskyrmions, in both thin films and bulk samples has been challenging to date. Here we apply magnetosensitive microscopy combined with electrical transport to directly detect the emergence of antiskyrmions in crystalline microstructures of $\mathrm{Mn}_{1.4} \mathrm{PtSn}$ at room temperature. We reveal the THE of antiskyrmions and demonstrate its tunability by means of finite sizes, field orientation, and temperature. Our atomistic simulations and experimental anisotropy studies demonstrate the link between antiskyrmions and a complex magnetism that consists of competing ferro- and antiferromagnetic as well as chiral exchange interactions.
\end{abstract}

\title{
MAIN TEXT
}

\section{INTRODUCTION}

The field of skyrmionics comprises new phases of magnetic systems, where the individual spins align as whirls [1-4], that hold the potential to advance the understanding of topology in condensed matter physics. Fundamentally, magnetic textures, such as skyrmions [3, 5], antiskyrmions [6, 7] (ASKs) and related skyrmionic systems [8 10], are distinguished by the relative rotation of the individual magnetic moments with respect to one another in the presence of a magnetically ordered background. This topologically protected state can be used to robustly carry and store high-density information at fast speed with low power consumption, as proposed in racetrack memory spintronic devices [11, 12]. Antiskyrmions (from hereon ASK) in particular promise high stability and enormous speed [13 18]. Solitary skyrmion states as well as crystal lattices of skyrmion systems have been reported for many magnetic crystals that break inversion symmetry such as bulk chiral magnetic compounds [5, 19], multilayer heterostructures [20, 21], thin films [22], oxides [23], and more recently tetragonal Heusler systems [6, 24]. While experimental observations from, e.g., Lorentz transmission electron microscopy [6, 25], neutron scattering [5] and magnetic force microscopy [26, 27] 
have shown great advancement over the last decades, detection by electrical means is required for the realization of energy efficient spintronic devices [11, 12], probabilistic [28] and neuromorphic computing [29]. Furthermore, a pivotal point for applications is the ability to control and scale skyrmionics [30, 31], or even to transform one species of magnetic texture into another in multiskyrmion systems [24, 32, 33].

The tetragonal Heusler compound $\mathrm{Mn}_{1.4} \mathrm{PtSn}$ [34] has recently gained a lot of interest as it is a host of distinct topological states. Most excitingly, an ASK lattice can be established at room temperature. Besides the high tunability of the Heusler compounds [27, 32, 35], the competition of magnetic exchange interactions and anisotropy lead to an enhanced temperature range over which ASKs can be stabilized in comparison to skyrmion bulk systems. Bulk $\mathrm{Mn}_{1.4} \mathrm{PtSn}$ displays a complex correlation of exchange between two Mn sublattices that leads to a spin reorientation from a collinear arrangement to a noncollinear at temperatures $T_{\mathrm{SR}} \approx T=170 \mathrm{~K}$ and below, which contributes to the anisotropy at low temperatures. At temperatures above the spin reorientation complex magnetic textures can be realized thanks to the combination of inplane (perpendicular to the $c$ axis) and anisotropic DzyaloshinskiiMoriya interaction (DMI), crystal anisotropy along the tetragonal axis, and dipole-dipole interaction due to large moments $\left(>4 \mu_{\mathrm{B}}\right)$. Above $T_{\mathrm{SR}}$ in the absence of an external magnetic field, the compound prefers a spin-spiral state that is directly connected to the ratio of the exchange interaction and DMI. For a finite range of external magnetic fields, a long-range (> $100 \mathrm{~nm}$ ) hexagonal ASK phase as well as a short-range noncoplanar state $(<1 \mathrm{~nm})$ were found [6]. Recently, new topological magnetic skyrmion-like textures have been proposed to exist that are tunable by direction of the applied magnetic field [24, 35].

The topological nature of skyrmions and ASKs should be observable in terms of a unique response to an external electric field, i.e., a topological Hall effect (THE) in the electrical transport. The THE belongs to the family of Berry curvature Hall effects [36, 37], where emergent fields due to a real-space variation of the local magnetism, describable by the Berry curvature [38], are the origin of a transverse voltage. This is sketched in Fig. 1a. Closely related to the THE is the anomalous Hall effect (AHE), due to the interaction of localized and conduction electrons [39]. In a finite magnetic field, the Hall response is a voltage transverse to the applied current. Phenomenologically, the total Hall effect is usually decomposed into three contributions [39]: The ordinary Hall effect (OHE) that scales with the external magnetic field, $\mathbf{H}$; The AHE, which scales with the magnetization $\mathbf{M}$, describable in terms 
of an intrinsic momentum-space Berry curvature and extrinsic scattering mechanisms, such as skew and side-jump scattering [39, 40]; The THE, which can be described in terms of the chiral product $\chi_{i j}=\mathbf{S}_{i} \cdot\left(\mathbf{S}_{j} \times \mathbf{S}_{k}\right)$, where $\mathbf{S}_{i}$ is the local spin orientation. The latter may be utilized to identify the sign of the topological charge, being opposite for skyrmions and ASKs, respectively.

In this work we successfully combined in-situ transport with magnetosensitive microscopy, which enables us to detect the negative THE right at the point, where the magnetic structure establishes a hexagonal ASK lattice, observable from $T_{\mathrm{SR}}$ to room temperature and above. In contrast to the general approach of micromagnetic simulations we apply atomistic spin dynamic calculations that capture the underlying complex magnetism of $\mathrm{Mn}_{1.4} \mathrm{PtSn}$, inevitably linked to the emergence of ASKs at high temperatures and skyrmions at low temperatures. We show how the periodicity of magnetic textures and the THE of ASKs scales with thickness. Our ferromagnetic resonance (FMR) studies reveal the distinct magnetic anisotropies above and below $T_{\mathrm{SR}}$ linked to the emergence of multiple skyrmionic textures.

\section{RESULTS AND DISCUSSION}

\section{Room-temperature THE signature of antiskyrmions}

In Figure 1a we show a schematic of a step-like device, where an ASK lattice is formed in an external magnetic field applied perpendicular to the $a b$ plane of the step device (for further details see Supplementary Notes S1-S3). ASKs possess an anisotropic winding with a topological charge of opposite sign as compared to isotropic Bloch and Néel skyrmion systems [7]. As the thickness, $d$, of a device is decreased the density of the ASK lattice increases, which in turn increases the magnitude of the topological charge that can directly be determined by a voltage, $V_{x y}$, transverse to the electric current, i.e., the topological Hall effect (THE). Figure 1b displays false-color scanning electron microscope images of two different devices fabricated by focussed-ion-beam (FIB) assisted patterning, namely, a step-like device with thicknesses $d=11.2,8.4$, and $2.7 \mu \mathrm{m}$ and a standalone device with $d=1.0 \mu \mathrm{m}$. The devices are set up as a Hall bar to simultaneously measure the Hall voltage while observing the magnetic state in-situ via polar magnetooptical Kerr effect (MOKE) microscopy [41]. In Figure 1c we display the total measured Hall effect for all thicknesses 
from the demagnetized state $\left(\mu_{0} H=0\right)$ up to a nearly saturated state $\left(H_{\mathrm{S}}\right)$. For $d<8.4 \mu \mathrm{m}$ a noticeable hysteresis appears in the Hall effect before saturation, which is due to a change in the magnetic texture. The inset of Fig. 1c displays a zoom in of the hysteresis for a $2.4 \mu \mathrm{m}$ thick device with the up (down) sweep direction denoted as black (red) arrows. The down sweep stays in the saturated state down to $430 \mathrm{mT}$ due to the anisotropy in the magnetic exchange interactions and the critical aspect ratio of the sample that adds an additional shape anisotropy contribution [27]. However, on the up sweep there are two clearly distinguishable slopes: The first originates from distinct conical magnetic spin-spirals formed by the two non-equivalent magnetic Mn sublattices [42]. This hysteresis is also discernible in the magnetization, as we show in Supplementary Note S5 The second, highlighted in yellow, shows a slope change and originates from the formation of the ASK lattice. The Hall effect in the inset of Fig. 1c is simultaneously measured with polar MOKE microscopy, shown in Fig. 1d as a function of external field. The MOKE images of Fig. 1d, congruent with Hall measurements, show a saturated magnetized state down to $430 \mathrm{mT}$, where magnetic spinspirals suddenly emerge. When increasing field from negative towards positive values the conical spin-spiral state starts to slowly nucleate individual ASKs and ASK strings. Then suddenly at $540 \mathrm{mT}$, an ASK lattice emerges, exactly where we observe the THE to emerge. A more refined series of MOKE images plus a video sequence that shows the full field range can be found in Supplementary Note S5 and Supplementary Movie S1.

The magnetic structure of $\mathrm{Mn}_{1.4} \mathrm{PtSn}$ and the expected transport signature in the ASK phase

In Figure 2a we sketch the spin configuration and the respective interactions present in $\mathrm{Mn}_{1.4} \mathrm{PtSn}$. The combination of DMI, induced by the D2d symmetry, FM and AFM interaction, is an ideal foundation for diverse magnetic textures [42]. We find that the physics of topological textures in $\mathrm{Mn}_{1.4} \mathrm{PtSn}$ can be accurately modeled purely from the exchange interactions and magnetocrystalline anisotropy in three atomic layers of the two magnetic sublattices [10, 15, 43. More significantly, the ratios of the exchange constants determine the underlying physics; therefore, we tuned the classical parameters in atomistic spin-dynamic simulations to clearly show the effects seen in the experiments. There are three sets of exchange interactions and DMI crucial for the stabilization of inplane ASKs 
in $\mathrm{Mn}_{1.4} \mathrm{PtSn}$. The first interaction, $J_{1}$ between the $\mathrm{Mn}(1)$ and $\mathrm{Mn}(2)$ sublattice, is the shortest magnetic distance and has the largest DMI. The second interaction, $J_{2}$ between magnetic sublattices of the same type and perpendicular to the $c$ axis, is ferromagnetic, i.e. the main interaction responsible for the Curie temperature. The interaction $J_{3}$ for the $\mathrm{Mn}$ sublattice $\mathrm{Mn}(2)$ at the $4 d$ Wyckoff position is of AFM nature, and assists in the formation of the noncoplanar structure below $T_{\mathrm{SR}}$. These AFM and FM interactions compete to form double chiral spin-spirals in order to minimize the energy. The chirality degeneracy is lifted by the DMI.

The calculated out-of-plane (inplane) component of the spin-spiral and ASK state is shown in the upper (lower) panel in Fig. 2b and C, respectively. The yielded magnetization hysteresis (Fig. 2d) is congruent with our experimental observations of a finite hysteresis away from zero field for a transition from the spin-spiral ground state into a stable ASK phase (for further details see Supplementary Note S6). The calculated magnetization exhibits a weak region, that is a shallow slope change right before the saturation field, consistent with our THE observations (see Fig. 1c). The chiral product, $\chi_{i j}$ leads to a topological winding number, $w=1 / 4 \pi \int \hat{\mathbf{M}} \cdot\left(\delta_{x}\left(\hat{\mathbf{M}} \times \delta_{y} \hat{\mathbf{M}}\right) d x d y\right.$, where $\delta_{i}=\delta / \delta_{i}$, and $\hat{\mathbf{M}}(r, t)=\mathbf{M}(r, t) /|\mathbf{M}|$ is the direction of the magnetization at each spatial position. The presence of $w$ causes an emergent magnetic field, $H_{z}^{e}=\frac{\hbar}{2} \epsilon_{z x y} \hat{\mathbf{M}} \cdot\left(\delta_{x} \hat{\mathbf{M}} \times \delta_{y} \hat{\mathbf{M}}\right)$, originating from a real-space Berry curvature [36]. The so-called topological charge, $q_{\mathrm{T}}$, is negative for skyrmions and positive for ASKs. Thereby, the THE is a direct link to the topology of the magnetic texture, $\rho_{x y}^{\mathrm{THE}}=$ $R_{x y}^{\mathrm{THE}} H^{e}$, where the Hall coefficient, $R_{x y}^{\mathrm{THE}}$, is tied to the complex multiorbital electronic structure 44]. The composite nature of $R_{x y}^{\mathrm{THE}}$ and $H^{e}$ complicates the differentiation of distinct skyrmionic configurations with distinct winding numbers across multiple materials. However, in $\mathrm{Mn}_{1.4} \mathrm{PtSn}$ distinct magnetic textures are induced by the external field.

In the lower panel of Fig. $2 \mathrm{~d}$ we plot $q_{\mathrm{T}}$ against the external field. $q_{\mathrm{T}}$ has an opposite sign as compared to the net charge in the low-field spin-spiral phase and only exists in the up sweep (orange curve). The down sweep (black curve) remains flat and only exhibits a positive contribution at lower fields, originating from the reestablishing spin-spiral phase. Hence, the subtraction of field-up and -down sweep mostly cancels out the component from the ordered phase and should, therefore, yield the THE due to ASKs directly. In Supplementary Note S7 we provide subtraction results from our experimental transport data at various temperatures. 


\section{Thickness and temperature dependent THE of ASKs}

While MOKE microscopy in combination with electrical transport allows for simultaneous measurements of the magnetic texture and the Hall effect in $\mathrm{Mn}_{1.4} \mathrm{PtSn}$, it is limited in spacial resolution by the wavelength of the optical light used [41]. We therefore use magnetic force microscopy (MFM) to resolve the ASK lattice induced in devices of different thickness d. Figure 3a shows MFM images at zero and $0.55 \mathrm{~T}$ for a $5 \mu \mathrm{m}$ and $1.5 \mu \mathrm{m}$ thick device, respectively. The spin-spiral domain bands and the ASK lattice are discernible with varying periodicity, depending on $d$ (the lower $d$ the smaller the magnetic periodicity). The fast Fourier transforms (FFTs), shown in the lower panels in Fig. 3a, highlight the change in topology as the system transforms from the spin-spiral into the hexagonal ASK phase. The

size and periodicity of the ASKs are affected by the thickness as has been shown recently-the latter varies almost linearly with thickness [27, 45]. This suggests that the overall THE (shown in Fig. 3b for various thicknesses) is expected to grow quadratically with decreasing $d$, as it is directly related to the ASK density. Excitingly, this trend is also confirmed by the enhanced THE component in thinner devices, highlighted by the fit (red dashed line) in Fig. 3c. Moreover, we find that the THE remains as pronounced as at room temperature all the way down to temperatures close to $T_{\mathrm{SR}}$ (see Fig. $3 \mathrm{~d}$ and Supplementary Note S7). Below $T_{\mathrm{SR}}$, it is strongly suppressed until it vanishes and additional step-like jumps in the Hall resistivity occur. The saturation field marks the upper boundary of the THE field region (yellow). With decreasing temperature, it shifts towards higher values until saturating near $T_{\mathrm{SR}}$, where noncoplanar magnetism starts to take over. Its overall amplitude starts to subside around $T_{\mathrm{SR}}$ as can be seen from Fig. 3e. Below $150 \mathrm{~K}$, we cannot unambiguously link the observed deviations to skyrmions. Further microscopy and spectroscopy studies are highly desirable in order to understand the details of the low-temperature behavior.

\section{Emergence of a new state below $T_{\mathrm{SR}}$}

Below $T_{\mathrm{SR}}$, the overall Hall response decreases more rapidly and the hysteresis in field narrows (see Fig. 4a). We present a detailed angular study on device B in Supplementary Note S8. As we tilt the magnetic field away from $H \| c$, the hysteresis subsides (it is absent in the high temperature curves shown in Fig. 4b). For temperatures near and below $T_{\mathrm{SR}}$ 
and the field being oriented away from the $c$ direction, we observe a prominent hump-like enhancement of $\rho_{x y}(H)$ close to the saturation field (see for example the maximum in $\rho_{x y}(H)$ at $180 \mathrm{~K}$ and $H \| b$ in Fig. 4b). This feature was already explored for bulk samples [34, 42]. Its origin is a THE linked to the establishment of a noncoplanar spin structure with a strong real-space Berry curvature. Excitingly, for fields along the $b$ axis, $\rho_{x y}(H)$ starts acquiring a negative high-field slope and even changes sign at low temperatures (Fig. 4b). At $2 \mathrm{~K}$, we observe a surprisingly large transverse transport signal even without any out-of-plane field component. For angles within only few degrees off $\theta=90^{\circ}$ it is accompanied by a new hysteresis that exhibits opposite sign as compared to the one for $H \| c$. As we finetune the angular step width, we observe a hysteresis that extends to fields even larger than $4 \mathrm{~T}$, being extremely sensitive to minute changes in $\theta$ (see Supplementary Note S8). Moreover, step-like changes in the hysteretic part of $\rho_{x y}(H)$ occur (see for example the $150 \mathrm{~K}$ curve in Fig. 4b). Hence, our data indicates an intriguing magnetic behavior for low temperature and field aligned within the ab planes, likely originating from the noncoplanar magnetism with a much stronger magnetocrystalline anisotropy for low temperatures. As we show in Fig. 4d and Supplementary Note S8, both the AHE and the OHE deviate from the conventional $\cos \theta$ dependence, observable at room temperature, as temperature is tuned to $T_{\mathrm{SR}}$ and below (see red dotted fits). This further indicates an enhanced saturation field for the inplane field orientation. These temperature- and angle-dependent changes are independent of the device thickness (confirmed for devices A, B, and C).

To further explore the temperature-dependent change in the magnetic exchange interactions we conducted fixed-frequency FMR experiments on a thin ( $800 \mathrm{~nm}$ ) lamella sample. In Fig. 4e we present FMR spectra recorded at $260 \mathrm{~K}$ and $10 \mathrm{~K}$ for two field directions each. The spectra were recorded while sweeping the field from the negative field-polarized state to the positive side and back, i.e. following the Hall-effect hysteresis curves. For further details, see also Supplementary Note S9. We also observe the hysteresis around $0.5 \mathrm{~T}$ for $H \| c$ in the FMR data (see black curve in Fig. 4e and insert). Furthermore, at $260 \mathrm{~K}$, we observe one narrow resonance mode attributed to the field-polarized state. As we tilt the field towards $H \| b$, the resonance preserves its narrow shape and the nearly isotropic angular dependence, varying between 0.62 and $0.75 \mathrm{~T}$. This suggests that above $T_{\mathrm{SR}}$, the magnetocrystalline anisotropy is weak. The maximum resonance field (corresponding to a magnetically hard axis) occurs around $24^{\circ}$. An approximate fit to the angle-dependent data yields a weak 
effective inplane-anisotropy field, which is comparable in magnitude to the ASK compound $\mathrm{Fe}_{1.9} \mathrm{Ni}_{0.9} \mathrm{Pd}_{0.2} \mathrm{P}$ [33]. At temperatures well below $T_{\mathrm{SR}}$, the main resonance mode becomes broad for $H \| c$ and asymmetric. In Fig. 4f the error bars represent the resonance linewidth (half width half maximum). Such a linewidth broadening is typical for the so-called field dragging, i.e., when $H \bigvee M$ due to a nonuniform magnetization for example. We observe that for high tilt angles close to the $b$ axis, resonances become narrower and shift to much lower fields of $0.1 \mathrm{~T}$. The overall angular dependence indicates that the anisotropy is much stronger below $T_{\mathrm{SR}}$. These significant changes in the magnetic anisotropies can explain the previously reported vanishing of ASKs and emergence of Bloch-type skyrmions at low temperatures [35]. In addition, the observed hysteresis in transport at high fields indicates new hard-magnetic behavior for the inplane-field orientation. This, therefore, may provide the right environment of the establishment of other distinct skyrmionics, e.g., antiferromagnetic Bimerons [46].

\section{CONCLUSION}

In sum, we demonstrated the direct detection of the THE of ASKs in the Heusler compound $\mathrm{Mn}_{1.4} \mathrm{PtSn}$ by combining electrical transport with magnetosensitive microscopy. Our studies of transport, magnetization and ferromagnetic resonance reveal a semi-hard-magnetic phase, and hence the unique link of ASKs to anisotropies, not possible in the cubic B20 compounds with weak anisotropy. We find this hard magnetic phase to increase as we reduce the thickness of the sample, which is particularly interesting for scalable electronic devices [47 49]. Many hard magnetic materials are strongly susceptible to finite sizes and may display nontrivial magnetic textures detectable by Hall transport [29, 50]. As the thickness is decreased the density of ASKs increases, which we observe directly using MFM, leading to an increase in the THE signal. The temperature dependence of the magnetic anisotropy, reflected in our Hall and FMR results, reveals the link to the crystal structure. Our atomistic model, solely based on the competing magnetic exchange interactions, captures the mechanism behind the emergence of ASKs in $\mathrm{Mn}_{1.4} \mathrm{PtSn}$. The novelty of tunability of texture sizes and the importance of the exchange interactions offers additional ways to manipulate and detect ASKs. Yet, smaller sizes and new materials with enhanced THE are desirable. Our observations offers a route to room-temperature skyrmionics applications based on multiple 
distinct types of skyrmionics textures.

\section{METHODS}

Experimental Design. The objectives of this study were to visualize the emergence of ASKs in mesoscale devices that allow a simultaneous investigation of the electrical transport under the influence of an external magnetic field and disentangle other contributions to the electrical transport signature related to the complex magnetism of the multiskyrmion host compound $\mathrm{Mn}_{1.4} \mathrm{PtSn}$. We conducted polar magnetooptical Kerr microscopy as well as magnetic force microscopy on micronsized samples fabricated by focussed-ion-beam assisted patterning. The former microscopy technique was successfully applied for the in-situ detection of Hall at room temperature. Furthermore, a detailed study of the topological-Hall signature depending on sample thickness, field orientation, and temperature was conducted. Chip-based FMR measurements depending on temperature, field, and angle were performed to obtain information on the ferromagnetic component of the complex magnetic background around temperatures at which the THE, associated with ASKs, is subsiding.

Crystal growth. Single-crystals were prepared by flux-growth technique showing no microtwinning confirmed by Laue diffraction. The details can be found elsewhere [34].

FIB microfabrication. We fabricated transport devices from high-quality single crystals of $\mathrm{Mn}_{1.4} \mathrm{PtSn}$ by the application of Ga or Xe FIB microstructuring, which enable highresolution investigations of anisotropic transport. FIB micromachining has already proven extremely powerful in various other metallic compounds. A detailed description of the fabrication is provided in Supplementary Note 1 and 2. Further details on FIB-patterned transport devices can be found in previous works of some of the authors [51, 52]. Thin (few microns thick) lamella-shaped slices of $\mathrm{Mn}_{1.4} \mathrm{PtSn}$ were separated with FIB and manually transferred ex-situ onto a sapphire substrate into a thin layer of insulating two-component epoxy. We then deposited a $100 \mathrm{~nm}$ thick gold (Au) layer on top in order to electrically connect the leads with the crystal. With the help of FIB we thereafter patterned the Au interfaces into separate terminals. In a next step, we removed the Au from the central area of the slice before cutting the lamellae into Hall-bar-shaped transport devices, highlighted by purple color in Fig. 1b. For device A, shown in Fig. 1b the thin slice of the crystal was polished stepwise to three different thicknesses by FIB before the transfer onto the substrate. 
This way a microstructure device was created with three 3-point hall-bar devices connected in series.

Magnetotransport measurements. We conducted electrical-transport measurements in a $14 \mathrm{~T}$ Quantum Design physical properties measurement system by applying a standard a.c. lock-in technique. A SynkTek multichannel lock-in amplifier as well as Zurich Instruments lock-in amplifiers were used. The current is directed through the FIB cut microstructures (see purple colored parts in Fig. 1b). Dimension of the devices were determined using a scanning electron microscope.

Magnetic force microscopy. MFM measurements were performed in two instruments. For room-temperature measurements without external fields we used the Park Systems NX10 with MFM probes from Nanosensors at lift heights between 100 and $150 \mathrm{~nm}$. For measurements under magnetic field, we used the AIST-NT SmartSPM 1000 with MFM probes from Nanosensors at lift heights between 250 and $350 \mathrm{~nm}$ together with a Nd-based permanent magnet. The magnetic flux strength was measured at the position of the sample with the MAGSYS HGM09s magnetometer. All data analysis was performed with the Gwyddion software.

Polar magnetooptical Kerr microscopy. Experiments were conducted with an AxioScope-type Carl Zeiss wide-field polarization microscope at room temperature. The sample was placed onto the pole of a solenoid-type, water cooled electromagnet with a maximum magnetic field of $1.5 \mathrm{~T}$ aligned along the $c$ axis. Special care was taken to remove parasitic Faraday contributions by the application of a motorized analyzer. Further details of the method can be found elsewhere [41].

Ferromagnetic Resonance. A broadband frequency vector network analyzer (VNA) FMR [53] was used to probe the resonance properties of a $\mathrm{Mn}_{1.4} \mathrm{PtSn}$ lamella (20 x 100 x 0.8$) \mu \mathrm{m}^{3}$ prepared by FIB. A $20 \mu \mathrm{m}$ wide coplanar waveguide CPW was used for the transmission in an impedance-matched $(50 \Omega) \mathrm{RF}$ chip-carrier design. The detection of the FMR modes was carried out via the change in the forward transmission parameter $S_{21}$ using Keysight N5225A VNA. Field- and temperature-dependent measurements were performed in an Attocube DRY 1100 cryostat with a split-coil magnet at constant temperature and fixed frequency of $20 \mathrm{GHz}$ using $10 \mathrm{dBm}$ microwave input power. At each field step, the real and imaginary components of the S21 parameter were recorded, using 100 times averaging. 
Numerical calculations. First-principle calculations were performed on $\mathrm{Mn}_{1.4} \mathrm{PtSn}$ by using the full-potential linearized augmented plane-wave code FLEUR following the work of Vir et al. [42] From this, exchange parameters were extracted where the largest interactions were $J_{1}, J_{2}, J_{3}$, and $D$. The atomistic spin calculations were performed in the code VAMPIRE for a lattice with 372 x 372 unit cells. For computation efficiency the Heisenberg exchange parameters are decreased $\left(J_{1}=10 \mathrm{meV}, J_{2}=12.0 \mathrm{meV}, J_{3}=-4 \mathrm{meV}\right)$ and the DMI magnitude increased $\left(D_{1}=2.0 \mathrm{meV}\right)$. The magnetocrystalline anisotropy is single axis and was chosen to be $0.5 \mu \mathrm{eV}$. To simulate the effect of the spin-reorientation transition, we set $J_{3}=-2 \mathrm{meV}$. We used the Landau-Lifshitz-Gilbert equation to study the spin dynamics of the system.

\section{ACKNOWLEDGEMENTS}

We would like to acknowledge K. Geishendorf from IFW for his help with transport-device fabrications. We furthermore thank A. P. Mackenzie from MPI CPfS and R. Huebner from IBC HZDR for their support and for granting access to their FIB infrastructure. We are thankful for the help of R. Narkovic and R. Illing from HZDR with the preparation of the substrate used in the FMR experiments.

\section{Funding:}

We acknowledge the support of the HLD at HZDR, member of the European Magnetic Field Laboratory (EMFL). B.E.Z.C. acknowledges support from the International Max Planck Research School for Chemistry and Physics of Quantum Materials (IMPRS-CPQM). P.M. and L.M.E. acknowledges support by the German Research Foundation (DFG) under Grants No. EN 434/38-1 and MI 2004/3-1 as well as EN 434/40-1 as part of the Priority Program SPP 2137 "Skyrmionics".

We acknowledge support by the Collaborative Research Center SFB 1143 (project-id 247310070) and the Würzburg-Dresden Cluster of Excellence on Complexity and Topology in Quantum Matter - ct.qmat (EXC 2147, project-id 390858490). P.J.W.M. acknowledges support by the European Research Council (ERC) under the European Union's Horizon 2020 research and innovation program (grant no. 715730, MiTopMat). 


\section{AUTHORCONTRIBUTIONS}

Single crystals growth and characterization: PV

FIB microstructure fabrication: TH

Magnetotransport experiments: MW, SH, MU, RS, TH

MFM measurements: BEZC, PM

MOKE microscopy: IS, RS

FMR measurements: FJTG, KL

First principle and atomistic spin dynamic simulations: JG

Supervision: TH, JW, CF, LE, RS, KL, STEBG, JG

Writing and editing of the original draft: All authors

\section{COMPETING INTERESTS:}

The authors declare no competing interests.

\section{DATA AND MATERIALS AVAILABILITY:}

All data supporting the findings of this study are included in the main text and Supplementary Information. Raw data in Ascii format will be provided by the corresponding author upon reasonable request.

\section{SUPPLEMENTARY MATERIALS}

In addition we provide the following supplementary material:

Supplementary Notes (1 to 9$)$

Supplementary Figures (S1 to S16)

Supplementary Movie S1 


\section{REFERENCES}

[1] Bogdanov, A. N. \& Yablonskii, D. A. Thermodynamically stable "vortices" in magnetically ordered crystals. the mixed state of magnets. Zh. Eksp. Teor. Fiz. 95, 178-182 (1989).

[2] Bogdanov, A. N. \& Hubert, A. Thermodynamically stable magnetic vortex states in magnetic crystals. J. Magn. Magn. Mater. 138, 255-269 (1994).

[3] Roessler, U. K., Bogdanov, A. N. \& Pfleiderer, C. Spontaneous skyrmion ground states in magnetic metals. Nature 442, 797-801 (2006).

[4] Back, C. et al. The 2020 skyrmionics roadmap. J. Phys. D: Appl. Phys. 53, 363001 (2020). URL https://doi.org/10.1088/1361-6463/ab8418.

[5] Mühlbauer, S. et al. Skyrmion lattice in a chiral magnet. Science 323, 915-919 (2009).

[6] Nayak, A. K. et al. Magnetic antiskyrmions above room temperature in tetragonal heusler materials. Nature 548, 561 (2017).

[7] Koshibae, W. \& Nagaosa, N. Theory of antiskyrmions in magnets. Nat. Commun. 7, 10542 (2021). URL https://doi.org/10.1038/ncomms10542.

[8] Rybakov, F. N., Borisov, A. B., Blügel, S. \& Kiselev, N. S. New type of stable particlelike states in chiral magnets. Phys. Rev. Lett. 115, 117201 (2015). URL https://link.aps.org/ doi/10.1103/PhysRevLett.115.117201.

[9] Everschor-Sitte, K., Masell, J., Reeve, R. M. \& Kläui, M. Perspective: Magnetic skyrmions - overview of recent progress in an active research field. J. Appl. Phys. 124, 240901 (2018). URL https://doi.org/10.1063/1.5048972.

[10] Göbel, B., Mertig, I. \& Tretiakov, O. A. Beyond skyrmions: Review and perspectives of alternative magnetic quasiparticles. Phys. Rep. 895, 1-28 (2021). URL https://www . sciencedirect.com/science/article/pii/S0370157320303525.

[11] Nagaosa, N. \& Tokura, Y. Topological properties and dynamics of magnetic skyrmions. Nat. Nanotechnol. 8, 899-911 (2013). URL https://doi.org/10.1038/nnano.2013.243.

[12] Parkin, S. \& Yang, S.-H. Memory on the racetrack. Nat. Nanotechnol. 10, 195-198 (2015). URL http://www .nature.com/doifinder/10.1038/nnano.2015.41. 
[13] Barker, J. \& Tretiakov, O. A. Static and dynamical properties of antiferromagnetic skyrmions in the presence of applied current and temperature. Phys. Rev. Lett. 116, 147203 (2016). URL https://link.aps.org/doi/10.1103/PhysRevLett.116.147203.

[14] Zhang, X., Zhou, Y. \& Ezawa, M. Magnetic bilayer-skyrmions without skyrmion hall effect. Nat. Commun. 7, 10293 (2016). URL https://doi.org/10.1038/ncomms10293.

[15] Akosa, C. A., Tretiakov, O. A., Tatara, G. \& Manchon, A. Theory of the topological spin hall effect in antiferromagnetic skyrmions: Impact on current-induced motion. Phys. Rev. Lett. 121, 097204 (2018). URL https://link.aps.org/doi/10.1103/PhysRevLett.121.097204.

[16] Shen, L. et al. Current-induced dynamics of the antiferromagnetic skyrmion and skyrmionium. Phys. Rev. Applied 12, 064033 (2019). URL https://link.aps.org/doi/10.1103/ PhysRevApplied.12.064033.

[17] Jin, C., Song, C., Wang, J. \& Liu, Q. Dynamics of antiferromagnetic skyrmion driven by the spin hall effect. Applied Physics Letters 109, 182404 (2016). URL https://doi.org/10. $1063 / 1.4967006$.

[18] Rosales, H. D., Cabra, D. C. \& Pujol, P. Three-sublattice skyrmion crystal in the antiferromagnetic triangular lattice. Phys. Rev. B 92, 214439 (2015). URL https://link.aps.org/ doi/10.1103/PhysRevB.92.214439.

[19] Neubauer, A. et al. Topological hall effect in the a phase of mnsi. Phys. Rev. Lett. 102, 186602 (2009). URL https://link.aps.org/doi/10.1103/PhysRevLett.102.186602.

[20] Yang, M. et al. Creation of skyrmions in van der waals ferromagnet fe3gete2 on (co/pd)n superlattice. Sci. Adv. 6 (2020). URL https://advances.sciencemag.org/content/6/36/ eabb5157, https://advances.sciencemag.org/content/6/36/eabb5157.full.pdf.

[21] Moreau-Luchaire, C. et al. Additive interfacial chiral interaction in multilayers for stabilization of small individual skyrmions at room temperature. Nat. Nanotechnol. 11, 444-448 (2016).

[22] Yu, X. Z. et al. Near room-temperature formation of a skyrmion crystal in thin-films of the helimagnet fege. Nat. Mater. 10, 106-109 (2011). URL https://doi.org/10.1038/ nmat2916.

[23] Seki, S., Yu, X. Z., Ishiwata, S. \& Tokura, Y. Observation of skyrmions in a multiferroic material. Science 336, 198-201 (2012). URL https://science.sciencemag.org/content/ 336/6078/198, https://science.sciencemag.org/content/336/6078/198.full.pdf. 
[24] Jena, J. et al. Elliptical bloch skyrmion chiral twins in an antiskyrmion system. Nat. Commun. 11, 1115 (2020). URL https://doi.org/10.1038/s41467-020-14925-6.

[25] Shimojima, T. et al. Nano-to-micro spatiotemporal imaging of magnetic skyrmion's life cycle. Science Advances 7 (2021). URL https://advances.sciencemag.org/content/7/25/ eabg1322. https://advances.sciencemag.org/content/7/25/eabg1322.full.pdf.

[26] Milde, P. et al. Unwinding of a skyrmion lattice by magnetic monopoles. Science 340, 10761080 (2013). URL https://science.sciencemag.org/content/340/6136/1076.

[27] Zuniga Cespedes, B. E., Vir, P., Milde, P., Felser, C. \& Eng, L. M. Critical sample aspect ratio and magnetic field dependence for antiskyrmion formation in $\mathrm{mn}_{1.4} \mathrm{PtSn}$ single crystals. Phys. Rev. B 103, 184411 (2021). URL https://link.aps.org/doi/10.1103/PhysRevB. 103.184411,

[28] Zázvorka, J. et al. Thermal skyrmion diffusion used in a reshuffler device. Nat. Naonotechnol. 14, 658-661 (2019). URL https://doi.org/10.1038/s41565-019-0436-8.

[29] Song, K. M. et al. Skyrmion-based artificial synapses for neuromorphic computing. Nat. Electron. 3, 148-155 (2020).

[30] Yu, X. Z. et al. Motion tracking of 80-nm-size skyrmions upon directional current injections. Sci. Adv. 6 (2020). URL https://advances.sciencemag.org/content/6/25/eaaz9744. https://advances.sciencemag.org/content/6/25/eaaz9744.full.pdf.

[31] Han, M.-G. et al. Scaling, rotation, and channeling behavior of helical and skyrmion spin textures in thin films of te-doped cu2oseo3. Sci. Adv. 6 (2020). URL https://advances.sciencemag.org/content/6/13/eaax2138 https://advances.sciencemag.org/content/6/13/eaax2138.full.pdf.

[32] Ma, T. et al. Tunable magnetic antiskyrmion size and helical period from nanometers to micrometers in a d2d heusler compound. Adv. Mater. 32, 2002043 (2020). URL https: //onlinelibrary.wiley.com/doi/abs/10.1002/adma.202002043.

[33] Karube, K. et al. Engineering chiral and topological orbital magnetism of domain walls and skyrmions. Nat. Mater. 20, 335-340 (2021). URL https://doi.org/10.1038/ s41563-020-00898-w.

[34] Vir, P. et al. Tetragonal superstructure of the antiskyrmion hosting heusler compound mn1.4ptsn. Chem. Mater. 31, 5876-5880 (2019). URL https://doi.org/10.1021/acs. chemmater.9b02013, https://doi.org/10.1021/acs.chemmater.9b02013. 
[35] Peng, L. et al. Controlled transformation of skyrmions and antiskyrmions in a noncentrosymmetric magnet. Nat. Nanotechnol. 15, 181-186 (2020).

[36] Lux, F. R., Freimuth, F., Blügel, S. \& Mokrousov, Y. Engineering chiral and topological orbital magnetism of domain walls and skyrmions. Commun. Phys. 1, 60 (2018). URL https://doi.org/10.1038/s42005-018-0055-y.

[37] Lux, F. R., Freimuth, F., Blügel, S. \& Mokrousov, Y. Chiral hall effect in noncollinear magnets from a cyclic cohomology approach. Phys. Rev. Lett. 124, 096602 (2020). URL https://link.aps.org/doi/10.1103/PhysRevLett.124.096602.

[38] Berry, M. V. Quantal phase factors accompanying adiabatic changes. Proceedings of the Royal Society of London. A. Mathematical and Physical Sciences 392, 45-57 (1984).

[39] Nagaosa, N., Sinova, J., Onoda, S., MacDonald, A. H. \& Ong, N. P. Anomalous hall effect. Rev. Mod. Phys. 82, 1539-1592 (2010). URL https://link.aps.org/doi/10.1103/RevModPhys. 82.1539 .

[40] Bruno, P., Dugaev, V. K. \& Taillefumier, M. Topological hall effect and berry phase in magnetic nanostructures. Phys. Rev. Lett. 93, 096806 (2004). URL https://link.aps.org/ doi/10.1103/PhysRevLett.93.096806.

[41] Soldatov, I. V. \& Schäfer, R. Advanced MOKE magnetometry in wide-field Kerr-microscopy. J. Appl. Phys. 122, 153906 (2017). URL http://aip.scitation.org/doi/10.1063/1. 5003719 .

[42] Vir, P. et al. Anisotropic topological hall effect with real and momentum space berry curvature in the antiskrymion-hosting heusler compound $\mathrm{mn}_{1.4} \mathrm{PtSn}$. Phys. Rev. B 99, 140406 (2019). URL https://link.aps.org/doi/10.1103/PhysRevB.99.140406.

[43] Caretta, L. et al. Fast current-driven domain walls and small skyrmions in a compensated ferrimagnet. Nat. Nanotechnol. 13, 1154-1160 (2018). URL https://doi.org/10.1038/ s41565-018-0255-3.

[44] Spencer, C. S. et al. Helical magnetic structure and the anomalous and topological hall effects in epitaxial b20 fe $\mathrm{f}_{-y} \mathrm{Co}_{y}$ Ge films. Phys. Rev. B 97, 214406 (2018). URL https: //link.aps.org/doi/10.1103/PhysRevB.97.214406.

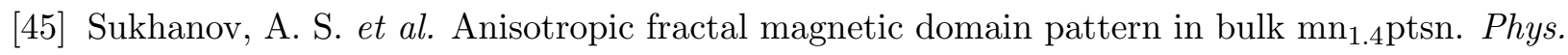
Rev. B 102, 174447 (2020). 
[46] Göbel, B., Mook, A., Henk, J., Mertig, I. \& Tretiakov, O. A. Magnetic bimerons as skyrmion analogues in in-plane magnets. Phys. Rev. B 99, 060407 (2019). URL https://link.aps. org/doi/10.1103/PhysRevB.99.060407.

[47] He, Y. et al. A new highly anisotropic rh-based heusler compound for magnetic recording. Adv. Mater. 32, 2004331 (2020). URL https://onlinelibrary.wiley.com/doi/abs/10. 1002/adma. 202004331.

[48] He, Y. et al. Topological hall effect arising from the mesoscopic and microscopic non-coplanar magnetic structure in mnbi (2020). Preprint, arXiv:2011.06340.

[49] $\mathrm{Wu}, \mathrm{H}$. et al. Observation of skyrmion-like magnetism in magnetic weyl semimetal co3sn2s2. Mater. Today Phys. 12, 100189 (2020). URL https://www.sciencedirect.com/science/ article/pii/S2542529320300134.

[50] Sokolov, D. A. et al. Metamagnetic texture in a polar antiferromagnet. Nat. Phys. 15, 671-677 (2019). URL https://doi.org/10.1038/s41567-019-0501-0.

[51] Moll, P. J. W. Focused ion beam microstructuring of quantum matter. Annu. Rev. Condens. Matter Phys. 9, 147-162 (2018). URL https://www.scopus.com/inward/record.uri?eid= $2-$ s2.0-85043606157\&doi=10.1146\%2fannurev-conmatphys-033117-054021\&partnerID= $40 \& m d 5=d f b 4 a a 0 a 4 d b f 8 e e 4 b e 8 f 7$ efd $6 \mathrm{~d} 622580$.

[52] Ronning, F. et al. Electronic in-plane symmetry breaking at field-tuned quantum criticality in CeRhIn5. Nature 548, 313-317 (2017). URL http://www.nature.com/doifinder/10. 1038/nature23315http://www.nature.com/articles/nature23315.

[53] Goncalves, F. J. T. et al. Collective resonant dynamics of the chiral spin soliton lattice in a monoaxial chiral magnetic crystal. Phys. Rev. B 95, 104415 (2017). URL https: //link.aps.org/doi/10.1103/PhysRevB.95.104415. 


\section{FIGURES}

Fig. 1. In-situ Hall effect in micro-fabricated $\mathbf{M n}_{1.4} \mathbf{P t S n}$ devices. (a) Schematic visualization of thickness-dependent ASKs induced by magnetic field, $H \| c$ axis, in a step-like sample with applied current along the $a$ axis. (b) False color SEM images of FIB-devices A and $\mathrm{C}$ with meander-shape (three thicknesses: 11.2, 8.4, and $2.7 \mu \mathrm{m}$ ) and Hall-bar geometry (thickness: $1.0 \mu \mathrm{m}$ ), respectively, contacted via sputter-deposited gold contacts. (For further details see Supplementare Notes S1-S3). (c) Hall-resistivity loop of samples A, B, and C for different thicknesses, $d$, along the $c$ direction with $H \| c$ at $T=300 \mathrm{~K}$. Inset: Reduced-area plot of the Hall-resistivity loop at $300 \mathrm{~K}$ for device $\mathrm{C}$ with $2.4 \mu \mathrm{m}$ thickness. Arrows indicate the field-sweep direction. Red dotted line is a linear fit. (d) Greyscale MOKE images for device $\mathrm{C}$ at three different fields for the up and down sweep, respectively.

Fig. 2. Magnetic structure of $\mathbf{M n}_{1.4} \mathbf{P t S n}$. (a) Sketch of the relevant interaction and coupling constants linking two Mn sublattices (red and blue). (b), (c) Atomistic spindynamic simulations for $H \| c$ : Color maps for an array of $(372 \times 372)$ unit cells showing the perpendicular z component in the spin-spiral and ASK phase, respectively. Black arrows represent the inplane component. The color scale is given in radiant, where $1 /-1$ corresponds to a spin alignment parallel to the normal. Lower panels show the inplane spin-winding component. (d) Upper panel: Calculated magnetization loop for fixed temperature given in meV; Lower panel: Calculated topological charge for opposite field-sweep directions (orange and black line).

Fig. 3. Thickness and temperature dependence of the magnetic textures and THE associated with ASKs. (a) MFM images (local magnetic z component represented by $\Delta \phi$ showing chiral domains and an ASK lattice for two samples prepared by FIB patterning with thicknesses of 5 and $1.5 \mu \mathrm{m}$. The data were recorded at room temperature in zero-field and at $550 \mathrm{mT}$ applied parallel to the $c$ axis, i.e., parallel to the line of sight. The lower panels show the respective FFTs (in arb. unit with logarithmic color scale). (b) THE component, $\rho_{x y}^{\mathrm{THE}}$, plotted against magnetic field for devices B, C, D, and E with thicknesses of $1,2.4,0.7$, and $0.8 \mu \mathrm{m}$, respectively. (c) Thickness dependence of $\rho_{x y}^{\mathrm{THE}}$ compared to the domain periodicity determined by recent MFM measurements [27, 32]. Dashed red line marks the expected size dependence. (d) $\rho_{x y}^{\mathrm{THE}}$ for temperatures between 10 and $300 \mathrm{~K}$ for device B with $d=1 \mu \mathrm{m}$. (e) Maximum amplitude of $\rho_{x y}^{\mathrm{THE}}$ plotted against the temperature. 
Fig. 4. Angle-dependent Hall and FMR at various temperatures. (a),(b) $\rho_{x y}(\mathrm{H})$ of device B with $1 \mu \mathrm{m}$ thickness recorded for $H \| c$ and $H \| a$, respectively, at various temperatures. (c) Extracted THE at three fixed temperatures for various angles. (d) Anomalous Hall coefficient extracted from linear fits to the high-field part, above $5 \mathrm{~T}$ of $\rho_{x y}(H)$ plotted against the tilt angle $\theta$. Red dotted lines are fits with $\rho_{x y}(H) \propto \cos \theta$. (e),(f) FMR forwardtransmission parameter, $S_{21}$. (e) FMR spectra for $H \| c$ and $H \| b$ recorded for a $0.8 \mu$ m thick lamella at 10 and 260 K. (f) Angular dependence of the FMR field, Error bars represent the resonance line-width values. 

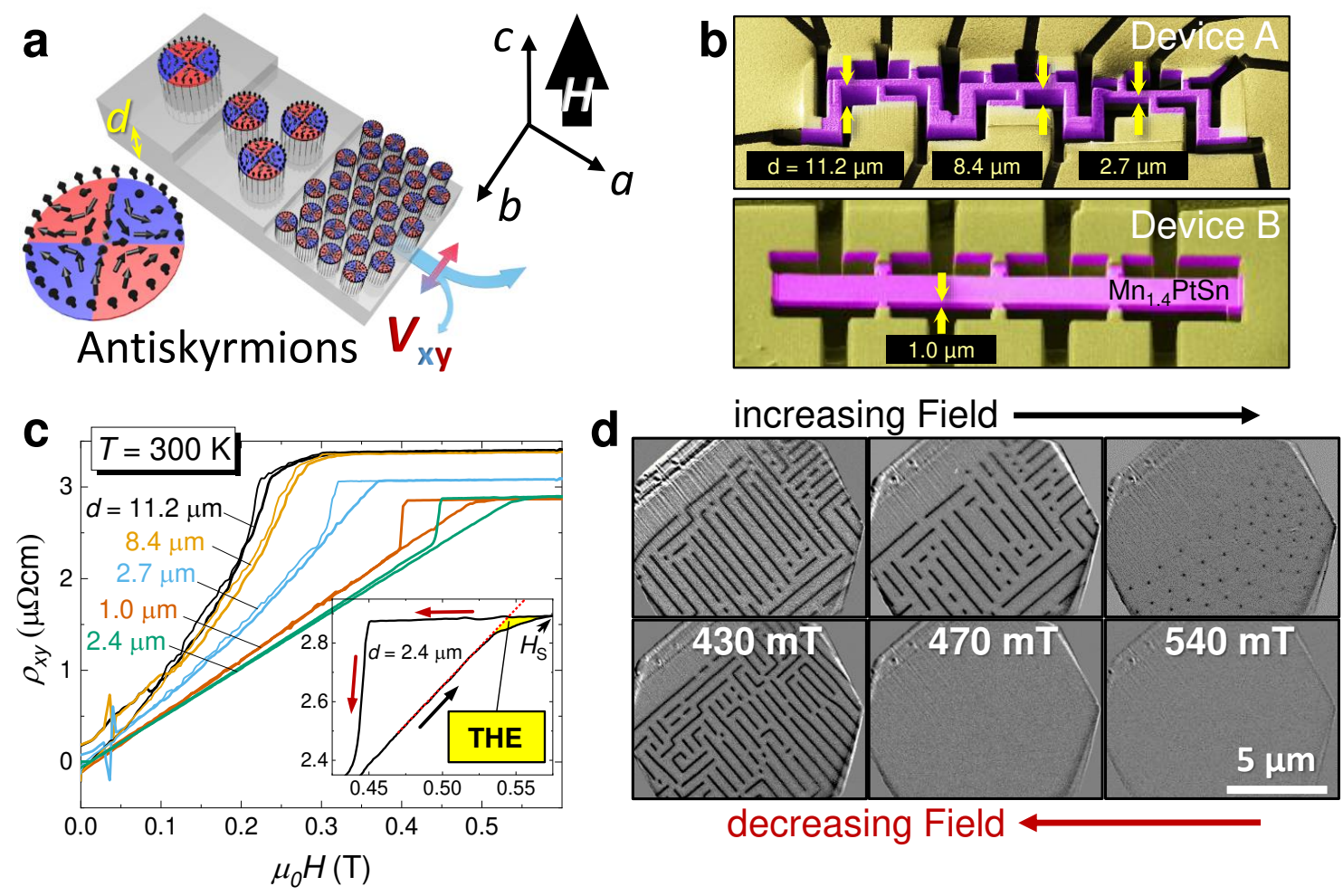

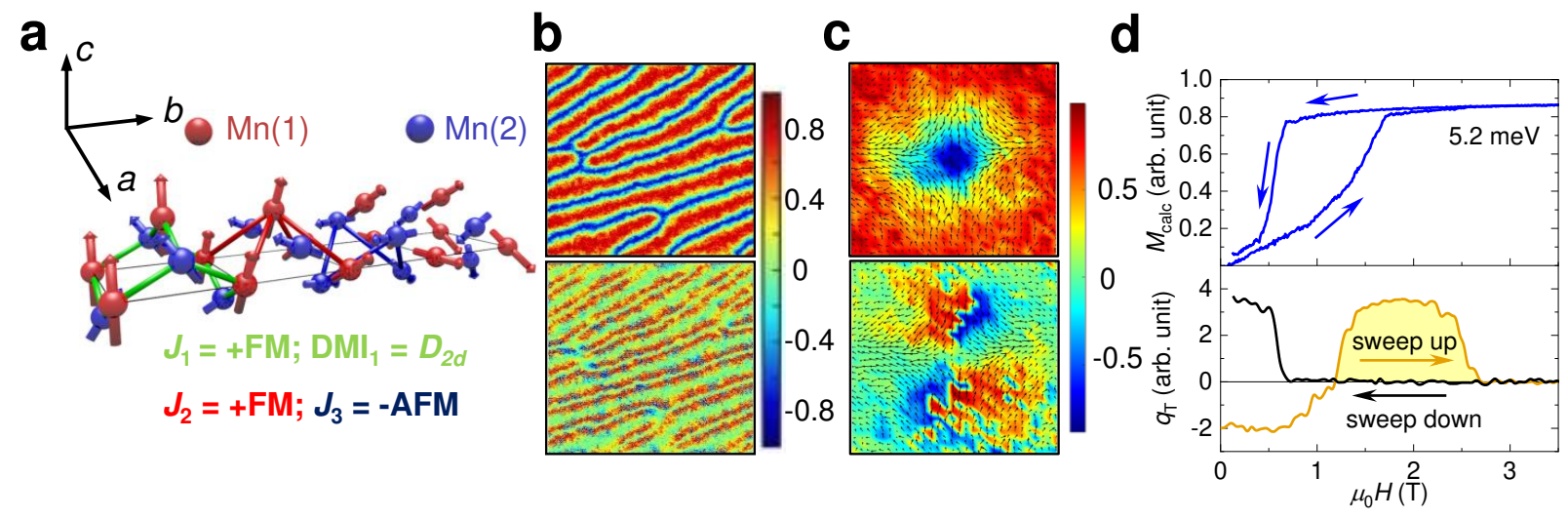


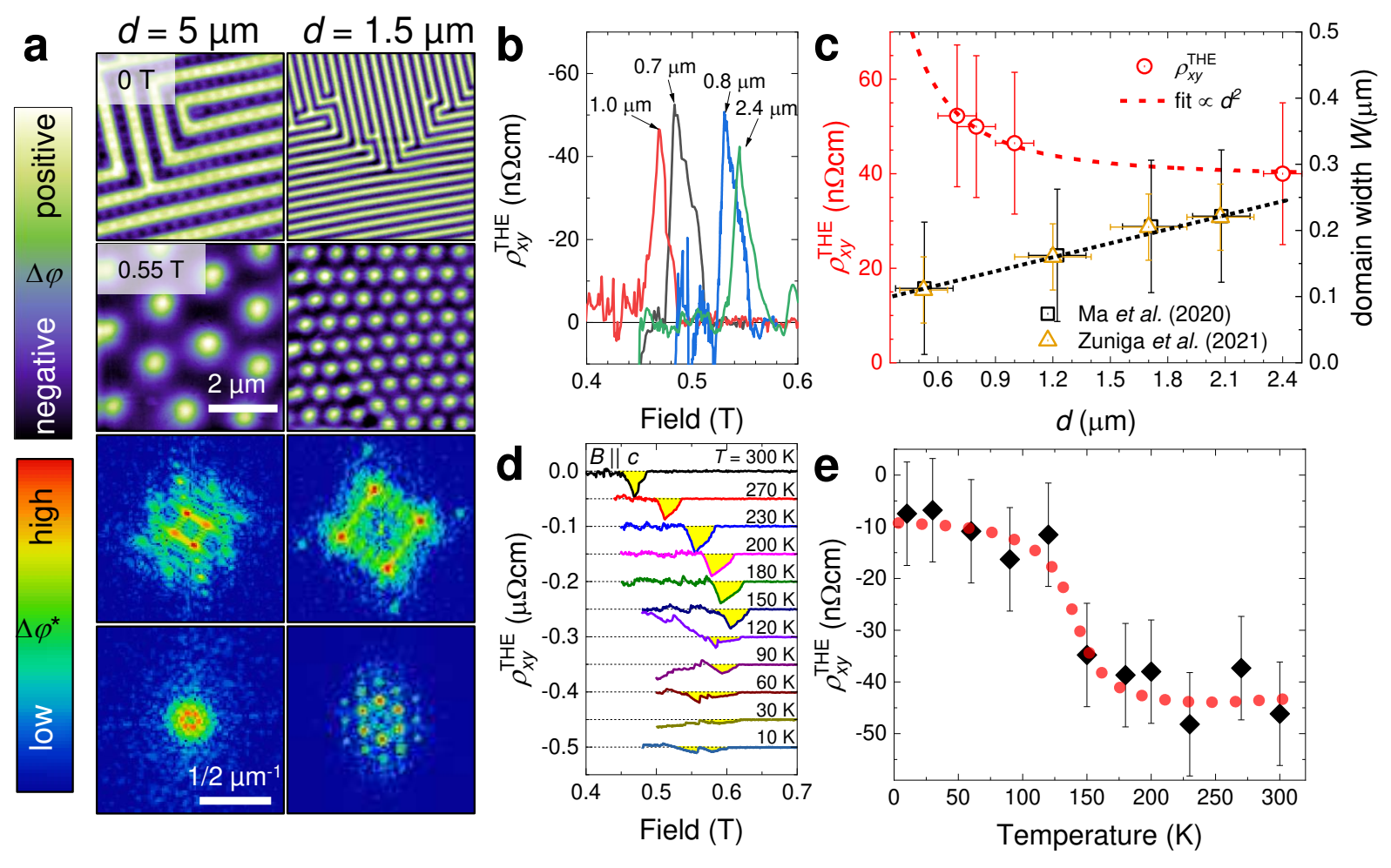



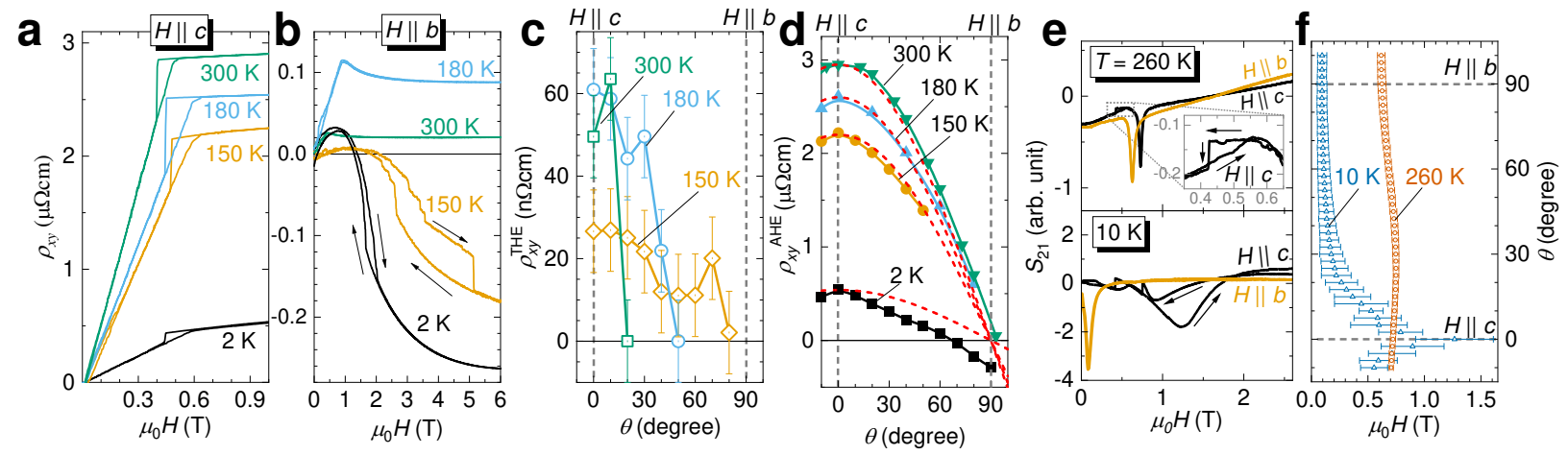

FIG. 4. 


\section{Figures}
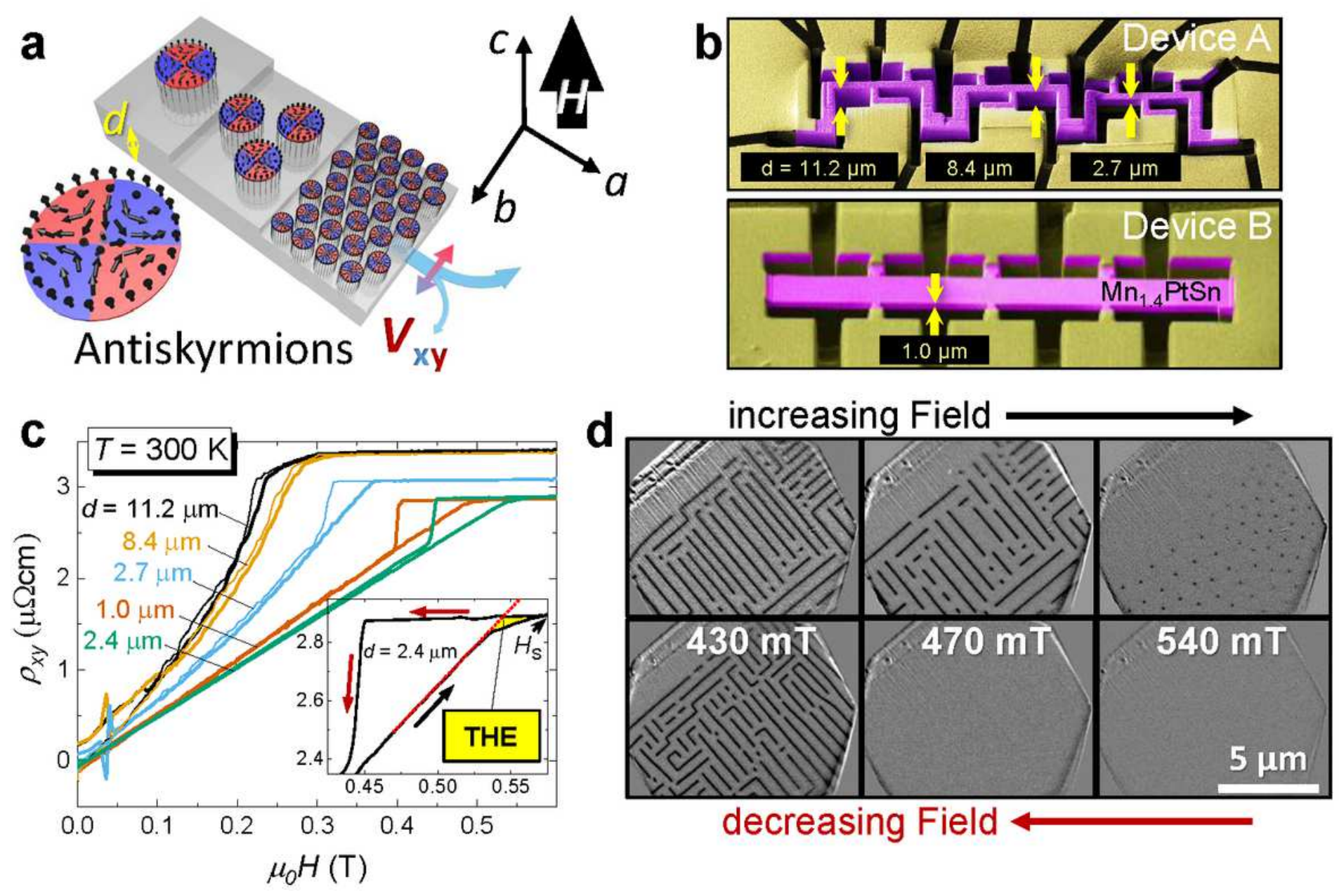

\section{Figure 1}

In-situ Hall effect in micro-fabricated Mn1.4PtSn devices. (a) Schematic visualization of thicknessdependent ASKs induced by magnetic field, $\mathrm{H} \| \mathrm{c}$ axis, in a step-like sample with applied current along the a axis. (b) False color SEM images of FIB-devices $A$ and $C$ with meander-shape (three thicknesses: 11.2, 8.4, and $2.7 \mu \mathrm{m}$ ) and Hall-bar geometry (thickness: $1.0 \mu \mathrm{m}$ ), respectively, contacted via sputter-deposited gold contacts. (For further details see Supplementare Notes S1-S3). (c) Hall-resistivity loop of samples A, $\mathrm{B}$, and $\mathrm{C}$ for different thicknesses, $\mathrm{d}$, along the $\mathrm{c}$ direction with $\mathrm{H} \| \mathrm{c}$ at $\mathrm{T}=300 \mathrm{~K}$. Inset: Reduced-area plot of the Hall-resistivity loop at $300 \mathrm{~K}$ for device $\mathrm{C}$ with $2.4 \mu \mathrm{m}$ thickness. Arrows indicate the field-sweep direction. Red dotted line is a linear fit. (d) Greyscale MOKE images for device $\mathrm{C}$ at three different fields for the up and down sweep, respectively. 
a



b

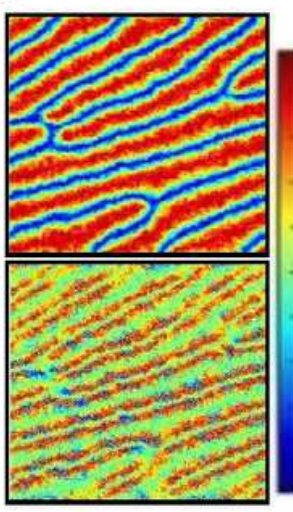

C



d

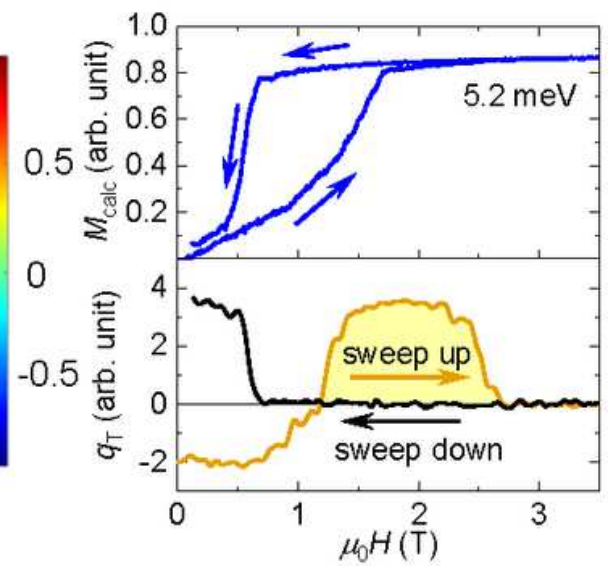

Figure 2

Magnetic structure of Mn1.4PtSn. (a) Sketch of the relevant interaction and coupling constants linking two Mn sublattices (red and blue). (b), (c) Atomistic spindynamic simulations for H\|c: Color maps for an array of $(372 \times 372)$ unit cells showing the perpendicular $z$ component in the spin-spiral and ASK phase, respectively. Black arrows represent the inplane component. The color scale is given in radiant, where $1 /-1$ corresponds to a spin alignment parallel to the normal. Lower panels show the inplane spin-winding component. (d) Upper panel: Calculated magnetization loop for fixed temperature given in meV; Lower panel: Calculated topological charge for opposite field-sweep directions (orange and black line).

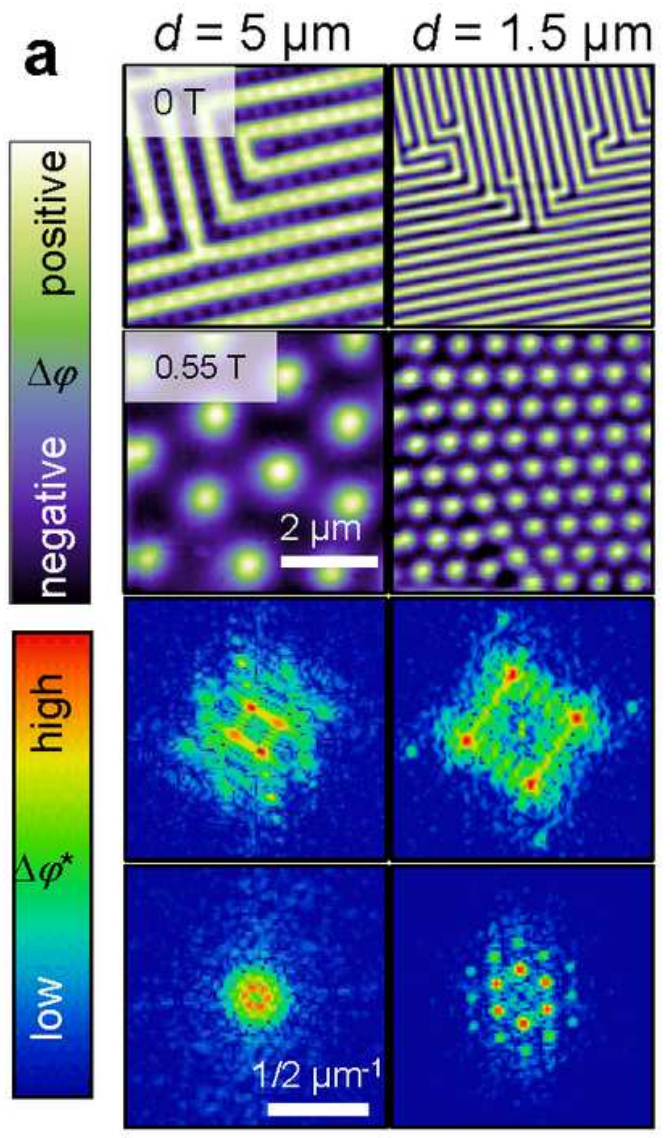

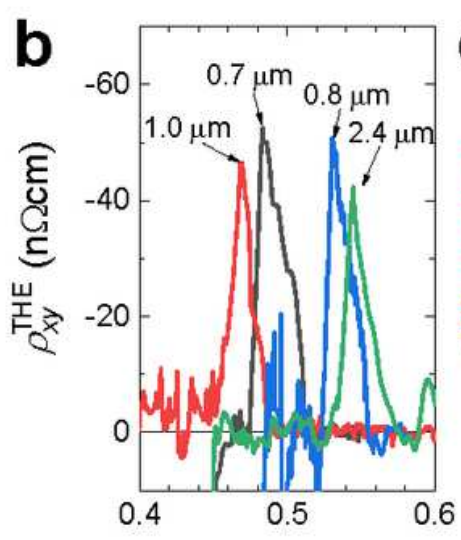

Field ( $\mathrm{T})$

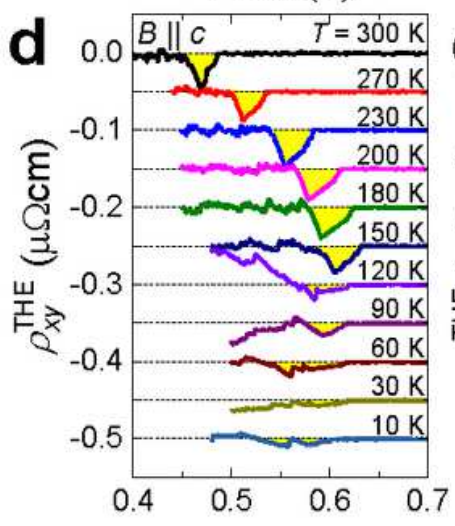

Field (T)



$d(\mu \mathrm{m})$

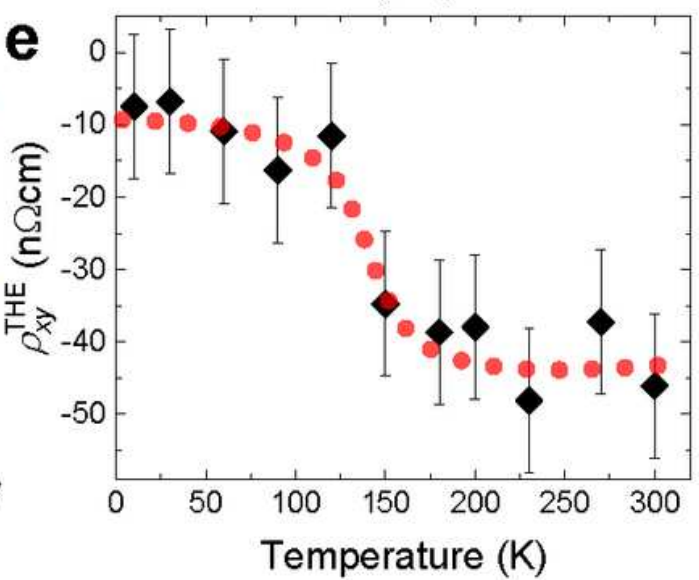




\section{Figure 3}

Thickness and temperature dependence of the magnetic textures and THE associated with ASKs. (a) MFM images (local magnetic z component represented by $\Delta \mathbb{Q}$ showing chiral domains and an ASK lattice for two samples prepared by FIB patterning with thicknesses of 5 and $1.5 \mu \mathrm{m}$. The data were recorded at room temperature in zero-field and at $550 \mathrm{mT}$ applied parallel to the $\mathrm{c}$ axis, i.e., parallel to the line of sight. The lower panels show the respective FFTs (in arb. unit with logarithmic color scale). (b) THE component, pTHE xy , plotted against magnetic field for devices $B, C, D$, and $E$ with thicknesses of 1, 2.4, 0.7, and 0.8 $\mu \mathrm{m}$, respectively. (c) Thickness dependence of $\rho T H E x y$ compared to the domain periodicity determined by recent MFM measurements [27, 32]. Dashed red line marks the expected size dependence. (d) $\rho$ THE xy for temperatures between 10 and $300 \mathrm{~K}$ for device B with $d=1 \mu \mathrm{m}$. (e) Maximum amplitude of $\rho$ THE xy plotted against the temperature.
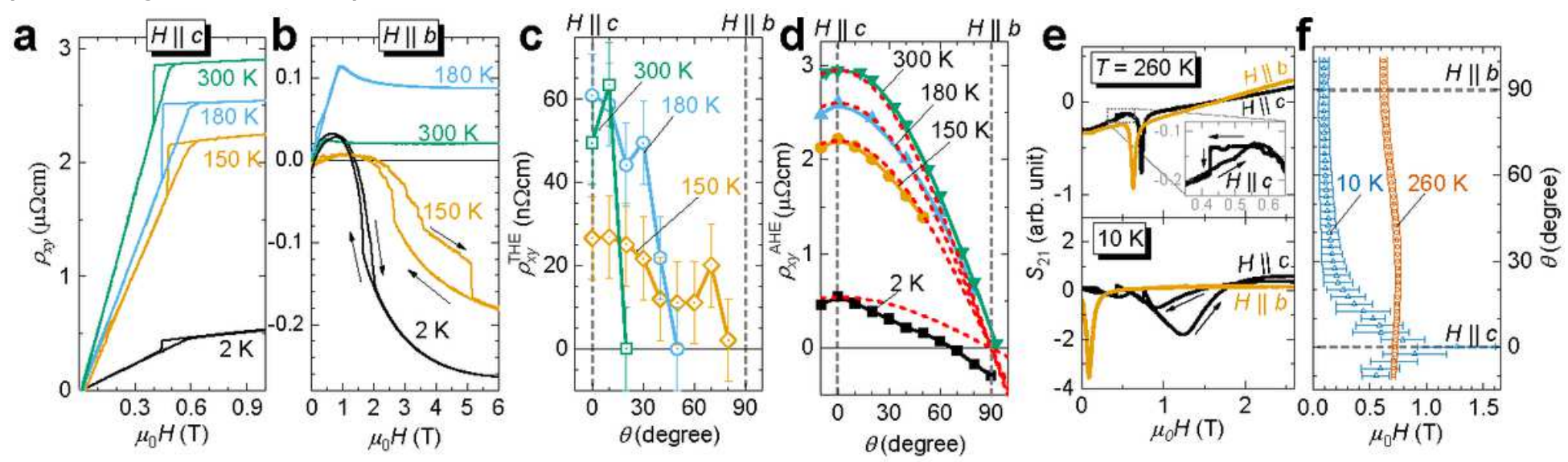

\section{Figure 4}

Angle-dependent Hall and FMR at various temperatures. (a),(b) $\rho x y(H)$ of device B with $1 \mu \mathrm{m}$ thickness recorded for $\mathrm{H} \| \mathrm{c}$ and $\mathrm{H} \| \mathrm{a}$, respectively, at various temperatures. (c) Extracted THE at three fixed temperatures for various angles. (d) Anomalous Hall coefficient extracted from linear fits to the high-field part, above 5T of $\rho x y(H)$ plotted against the tilt angle $\theta$. Red dotted lines are fits with $\rho x y(H) \otimes \cos \theta$. (e), (f) FMR forwardtransmission parameter, S21. (e) FMR spectra for $\mathrm{H} \| \mathrm{c}$ and $\mathrm{H} \| \mathrm{b}$ recorded for a $0.8 \mu \mathrm{m}$ thick lamella at 10 and $260 \mathrm{~K}$. (f) Angular dependence of the FMR field, Error bars represent the resonance line-width values.

\section{Supplementary Files}

This is a list of supplementary files associated with this preprint. Click to download.

- HelmSupplementOct2021v3.pdf

- Video1DeviceE0p8umfullsweep.mp4 